Research Article

\title{
Optimal Bounds for the Variance of Self-Intersection Local Times
}

\author{
George Deligiannidis ${ }^{1}$ and Sergey Utev $^{2}$ \\ ${ }^{1}$ Department of Statistics, University of Oxford, 24-29 St. Giles, Oxford OX1 3LB, UK \\ ${ }^{2}$ Department of Mathematics, University of Leicester, Leicester LE1 7RH, UK
}

Correspondence should be addressed to George Deligiannidis; deligian@stats.ox.ac.uk

Received 22 March 2016; Revised 17 May 2016; Accepted 7 June 2016

Academic Editor: Onesimo Hernandez-Lerma

Copyright (C) 2016 G. Deligiannidis and S. Utev. This is an open access article distributed under the Creative Commons Attribution License, which permits unrestricted use, distribution, and reproduction in any medium, provided the original work is properly cited.

For a $\mathbb{Z}^{d}$-valued random walk $\left(S_{n}\right)_{n \in \mathbb{N}_{0}}$, let $l(n, x)$ be its local time at the site $x \in \mathbb{Z}^{d}$. For $\alpha \in \mathbb{N}$, define the $\alpha$-fold selfintersection local time as $L_{n}(\alpha):=\sum_{x} l(n, x)^{\alpha}$. Also let $L_{n}^{\mathrm{SRW}}(\alpha)$ be the corresponding quantities for the simple random walk in $\mathbb{Z}^{d}$. Without imposing any moment conditions, we show that the variance of the self-intersection local time of any genuinely $d$-dimensional random walk is bounded above by the corresponding quantity for the simple symmetric random walk; that is, $\operatorname{var}\left(L_{n}(\alpha)\right)=O\left(\operatorname{var}\left(L_{n}^{\mathrm{SRW}}(\alpha)\right)\right)$. In particular, for any genuinely $d$-dimensional random walk, with $d \geq 4$, we have $\operatorname{var}\left(L_{n}(\alpha)\right)=O(n)$. On the other hand, in dimensions $d \leq 3$ we show that if the behaviour resembles that of simple random walk, in the sense that $\liminf _{n \rightarrow \infty} \operatorname{var}\left(L_{n}(\alpha)\right) / \operatorname{var}\left(L_{n}^{\mathrm{SRW}}(\alpha)\right)>0$, then the increments of the random walk must have zero mean and finite second moment.

\section{Introduction and Main Results}

Let $X, X_{1}, X_{2}, \ldots$ be independent, identically distributed, $\mathbb{Z}^{d}$ valued random variables, and define the random walk $S_{0}:=0$, $S_{n}=\sum_{j=1}^{n} X_{j}$, for $n \geq 1$. The special case with $\mathbb{P}\left(X_{i}=e\right)=$ $1 /(2 d)$, for all $e \in \mathbb{Z}^{d}$ with $|e|=1$, is known as the simple random walk in $\mathbb{Z}^{d}$ and will be denoted by $\left(\mathrm{SRW}_{n}\right)_{n \in \mathbb{N}_{0}}$.

Let $l(n, x)=\sum_{j=1}^{n} \mathbb{1}\left(S_{j}=x\right)$ be the local time of $\left(S_{n}\right)_{n \in \mathbb{N}_{0}}$ at the site $x \in \mathbb{Z}^{d}$, and define for a positive integer $\alpha$ the $\alpha$-fold self-intersection local time

$$
\begin{aligned}
L_{n} & =L_{n}(\alpha)=\sum_{x \in \mathbb{Z}^{d}} l(n, x)^{\alpha} \\
& =\sum_{i_{1}, \ldots, i_{\alpha}=0}^{n} \mathbb{1}\left(S_{i_{1}}=\cdots=S_{i_{\alpha}}\right) .
\end{aligned}
$$

We will denote the corresponding quantities for simple random walk in $\mathbb{Z}^{d}$ by $L_{n}^{\mathrm{SRW}}(\alpha, d)$ or simply $L_{n}^{\mathrm{SRW}}(\alpha)$ when the dimension is clear from the context.
Let $R^{+}$and $R^{-}$be, respectively, the semigroup and the group generated by the support of $X$,

$$
\begin{aligned}
R^{+} & :=\left\{x \in \mathbb{Z}^{d} \mid \mathbb{P}\left(S_{n}=x\right)>0 \text { for some } n \geq 0\right\}, \\
\bar{R} & :=\left\{x \in \mathbb{Z}^{d} \mid x=y-z \text { for some } x, y \in R^{+}\right\} .
\end{aligned}
$$

Following Spitzer [1], we call the random variable $X$ and the random walk it generates genuinely d-dimensional if the group $\bar{R}$ is $d$-dimensional.

The quantity $L_{n}(\alpha)$ has received considerable attention in the literature due to its relation to self-avoiding walks and random walks in random scenery. In particular let the random scenery $\left\{\xi_{x}, x \in \mathbb{Z}^{d}\right\}$ be a collection of i.i.d. random variables, independent of $\left(S_{n}\right)_{n}$, and define the process $Z_{0}=$ $0, Z_{n}=\sum_{i=1}^{n} \xi_{S_{i}}$. Then $\left(Z_{n}\right)_{n}$ is commonly referred to as random walk in random scenery and was introduced in Kesten and Spitzer [2], where functional limit theorems were obtained for $Z_{[n t]}$ under appropriate normalization for the case $d=1$. The case $d=2$, with $X_{i}$ centered with nonsingular covariance matrix, was treated in [3] where it 
was shown that $Z_{[n t]} / \sqrt{n \log n}$ converges weakly to Brownian motion. As is obvious from the identities $Z_{n}=\sum_{x \in \mathbb{Z}^{d}} l(n, x) \xi_{x}$ and $\operatorname{var}\left(Z_{n}\right)=\operatorname{var}\left[L_{n}(2)\right] \operatorname{var}\left(\xi_{x}\right)$, limit theorems for $\left(Z_{n}\right)_{n}$ usually require asymptotic results for the local times of the random walk $\left(S_{n}\right)_{n}$.

Such asymptotic results are usually obtained from Fourier techniques applied to the characteristic function $f(t)=$ $\mathbb{E}[\exp (\mathrm{it} \cdot X)]$, under the additional assumption of a Taylor expansion of the form $f(t)=1-\langle\Sigma t, t\rangle+o\left(|t|^{2}\right)$, where $\Sigma$ is a positive definite covariance matrix [3-7], which further requires that $\mathbb{E}|X|^{2}<\infty$ and $\mathbb{E} X=0$. Similar restrictions are also required for the application of local limit theorems such as in $[8,9]$.

In this paper, motivated by the results of Spitzer [1] for genuinely $d$-dimensional random walks and the approach of Becker and König [10], we will study the asymptotic behavior of $\operatorname{var}\left(L_{n}(\alpha)\right)$ without imposing any moment assumptions on the random walk. The central idea behind our approach is to compare the self-intersection local times $L_{n}(\alpha)$ of a general $d$-dimensional walk with those of its symmetrised version. In addition we will compare the self-intersection local times of a general $d$-dimensional random walk with those of the $d$-dimensional simple symmetric random walk, $\left(\mathrm{SRW}_{n}\right)_{n \in \mathbb{N}_{0}}$. It is well known that, for some positive constants $K_{\alpha, d}, \operatorname{var}\left(L_{n}^{\mathrm{SRW}}(\alpha, d)\right) \sim K_{\alpha, d} v_{d, \alpha}(n)$ as $n \rightarrow \infty$, for

$$
\begin{aligned}
& v_{1, \alpha}(n):=n^{1+\alpha}, \\
& v_{2, \alpha}(n):=n^{2} \log (n)^{2 \alpha-4}, \\
& v_{3, \alpha}(n):=n \log (n), \\
& v_{d, \alpha}(n):=n, \quad d \geq 4 .
\end{aligned}
$$

Several other cases have been treated in the literature, using a variety of methods.

A careful look at the literature reveals that the most difficult case in $d=2$ is the near transient recurrent case, where $\mathbb{P}\left(S_{n}=0\right) \sim C / n$, which corresponds to genuinely 2dimensional symmetric recurrent random walks, which will be referred to as a critical case. Surprisingly enough, the variance of the self-intersection local times in the critical case is asymptotically the largest.

Theorem 1. Let $X, X_{1}, X_{2}, \ldots$ be independent, identically distributed, and genuinely $d$-dimensional $\mathbb{Z}^{d}$-valued random variables, for any $d \geq 1$. Then, there exist positive constants $C_{\alpha, X}>c_{\alpha, X}>0$, depending on $\alpha$ and the distribution of $X$, such that for all $n$ large enough

$$
\operatorname{var}\left(L_{n}(\alpha)\right) \leq c_{\alpha, X} \operatorname{var}\left(L_{n}^{S R W}(\alpha, d)\right) \leq C_{\alpha, X} v_{d, \alpha}(n) .
$$

The result was motivated by $[1,10]$ and improves related results of Becker and König for $d=3$ and $d=4$. Several cases treated in $[3,4,10-13]$ can then be obtained as particular cases.

Moreover, we also show the surprising converse. More precisely, we show that the right asymptotic behaviour of $\operatorname{var}\left(L_{n}\right)$ implies that the jumps must have zero mean and finite second moment.
Theorem 2. Let $X, X_{1}, X_{2}, \ldots$, be independent, identically distributed, and genuinely $d$-dimensional with $d \leq 3$. If

$$
\liminf _{n \rightarrow \infty} \frac{\operatorname{var}\left(L_{n}(\alpha)\right)}{\operatorname{var}\left(L_{n}^{S R W}(\alpha)\right)}>0,
$$

then $\mathbb{E}|X|^{2}<\infty$ and $\mathbb{E} X=0$.

As it follows from Theorem 3 given below for $d=2,3$ and from Theorem 5.2.3 in Chen [12] for $d=1$, if $\mathbb{E} X=0$ and $0<\mathbb{E}|X|^{2}<\infty$, then $\liminf _{n} \operatorname{var}\left(L_{n}(\alpha)\right) / v_{d, \alpha}(n)>0$.

For any genuinely $d$-dimensional random walk with finite second moments and zero mean, the asymptotic behaviour of $\operatorname{var}\left(L_{n}(\alpha)\right)$ is similar to that of the $d$-dimensional simple symmetric random walk. Also, as it follows from our general bounds (see Proposition 4 and Corollary 7) that the asymptotic results for the genuinely $d$-dimensional random walk can be reproduced by those of the symmetric one-dimensional random walk with appropriately chosen heavy tails, as was indicated by Kesten and Spitzer [2]. The proofs are based on adapting the Tauberian approach developed in [13].

Theorem 3. Let $d=1,2,3$, and suppose that for $t \in \Gamma:=$ $[-\pi, \pi]^{d}$ one has

$$
\begin{aligned}
f(t) & =1-\gamma|t|+R(t), \quad \text { for } d=1, \\
\text { or } f(t) & =1-\langle\Sigma t, t\rangle+R(t), \quad \text { for } d=2,3,
\end{aligned}
$$

where $\sum$ is a nonsingular covariance matrix and $R(t)=o(|t|)$ for $d=1$ and $o\left(|t|^{2}\right)$ for $d=2,3$ as $t \rightarrow 0$. Then

$$
\begin{aligned}
& \operatorname{var}\left(L_{n}(\alpha)\right) \\
& \sim \begin{cases}\frac{\left(\pi^{2}+6\right)}{12} \frac{(\alpha !)^{2}(\alpha-1)^{2}}{(\gamma \pi)^{2 \alpha-2}} n^{2} \log (n)^{2 \alpha-4}, & \text { for } d=1, \\
\frac{(\alpha !)^{2}(\alpha-1)^{2}}{2(2 \pi \sqrt{|\Sigma|})^{2 \alpha-2}} n^{2} \log (n)^{2 \alpha-4}(\kappa+1), & \text { for } d=2, \\
\left(\kappa_{1}+\kappa_{2}\right) n \log n, & \text { for } d=3, \alpha=2,\end{cases}
\end{aligned}
$$

where

$$
\begin{aligned}
\kappa & :=\iint_{0}^{\infty} \mathrm{d} r \mathrm{~d} s\left[(1+r)(1+s) \sqrt{(1+r+s)^{2}-4 r s}\right]^{-1} \\
& -\frac{\pi^{2}}{6}
\end{aligned}
$$

and $\kappa_{1}$ and $\kappa_{2}$ are defined in (58) and (63), respectively.

Moreover, if $L^{\prime}(n, \alpha)$ is the self-intersection local time of another random walk, independent of $\left(S_{n}\right)_{n}$, whose characteristic function also satisfies (6), then $\operatorname{var}\left(L_{n}^{\prime}(\alpha)\right)=\operatorname{var}\left(L_{n}(\alpha)\right)(1+$ $o(1))$.

\section{Proofs}

2.1. General Bounds. We first develop a technique to treat random walks with independent but not necessarily identically distributed increments. 
Proposition 4 (general upper bound). Assume that $X_{1}$, $X_{2}, \ldots$ are independent $\mathbb{Z}^{d}$-valued random variables and let $S_{u, v}:=X_{u}+\cdots+X_{u+v}$. Suppose further that for all $n \in \mathbb{N}$ and integers $a, u, b, v \geq 0$, with $a+u \leq b$ and any $x \in \mathbb{Z}^{d}$, one has

$$
\begin{array}{r}
\mathbb{P}\left(S_{a, u} \pm S_{b, v}=x\right) \leq \phi(u+v), \\
\mathbb{P}\left(S_{a, u}=0\right)-\mathbb{P}\left(S_{a, u}+S_{b, v}=0\right) \leq \psi(u, v),
\end{array}
$$

where $\phi(u)$ is nonincreasing and $\psi(u, v)$ is nonincreasing in $u$ and is nondecreasing and subadditive in $v$ in the sense that $\psi(u, v+w) \leq A_{\psi}[\psi(u, v)+\psi(u, w)]$, for some constant $A_{\psi}$ independent of $u, v$, and $w$. Then, for some constant $K=$ $c A_{\psi}\left(1+A_{\psi}\right)^{\alpha-2}$ depending only on $\alpha$

$$
\begin{aligned}
& \operatorname{var}\left(L_{n}(\alpha)\right) \leq K n\left(\sum_{i=0}^{n-1} \phi(i)\right)^{2 \alpha-4} \\
& \quad \cdot \sum_{i, j, k=0}^{n-1}[\phi(j \vee i) \phi(k \vee i)+\phi(j) \psi(i+k, j)] .
\end{aligned}
$$

Proof of Proposition 4. We first write out the variance as a sum

$$
\operatorname{var}\left(L_{n}(\alpha)\right)=(\alpha !)^{2}
$$

$$
\begin{aligned}
& \cdot \sum_{k_{1} \leq \cdots \leq k_{\alpha}} \sum_{l_{1} \leq \cdots \leq l_{\alpha}}\left(\mathbb{P}\left[S_{k_{1}}=\cdots=S_{k_{\alpha}}, S_{l_{1}}=\cdots=S_{l_{\alpha}}\right]\right. \\
& \left.-\mathbb{P}\left[S_{k_{1}}=\cdots=S_{k_{\alpha}}\right] \mathbb{P}\left[S_{l_{1}}=\cdots=S_{l_{\alpha}}\right]\right) .
\end{aligned}
$$

An important role is played by the manner in which the two sequences are interlaced, since, for example, if $k_{\alpha} \leq l_{1}$ or $l_{\alpha} \leq$ $k_{1}$, the term vanishes by the Markov property.

We will treat the sum over indices with $k_{1} \leq l_{1}$. The sum over the remaining index set with $k_{1}>l_{1}$ can be treated in a similar fashion and will contribute a constant factor. Therefore, we assume that $k_{1} \leq l_{1}$ and we arrange the two sequences in an ordered sequence of combined length $2 \alpha$ which we denote as $\left(p_{1}, \ldots, p_{2 \alpha}\right)$; we also define $\left(\epsilon_{1}, \ldots, \epsilon_{2 \alpha}\right)$ where $\epsilon_{i}=0$ if $p_{i}$ came from $\mathbf{k}:=\left\{k_{1}, \ldots, k_{\alpha}\right\}$ and $\epsilon_{i}=1$ if $p_{i}$ came from $\mathbf{l}:=\left\{l_{1}, \ldots, l_{\alpha}\right\}$. Finally we define two new sequences $m_{0}, m_{1}, \ldots, m_{2 \alpha-1}$, and $\delta_{1}, \ldots, \delta_{2 \alpha-1}$, where $m_{0}:=$ $p_{1}, m_{i}=p_{i+1}-p_{i}$, and $\delta_{i}=\epsilon_{i+1}-\epsilon_{i}$, for $i=1, \ldots, 2 \alpha-1$. Notice that since we assume that $k_{1} \leq l_{1}$, we have $p_{1}=k_{1}$ and $\epsilon_{1}=0$. Let $v(\delta):=\sum_{i=1}^{2 \alpha-1}\left|\delta_{i}\right|$ denote the interlacement index. The terms with $v=1$ vanish, while the terms with $v=2$ will be considered separately.

Terms with $v \geq 3$. We first consider the sum $I_{n}$ over the terms with $v \geq 3$ for which we drop the negative part and obtain the bound

$$
\begin{aligned}
I_{n} & :=\sum_{\substack{k_{1} \leq \cdots \leq k_{\alpha} \\
l_{1} \leq \cdots \leq l_{\alpha} \\
k_{1} \leq l_{1}, v(\delta) \geq 3}} \mathbb{P}\left[S_{k_{1}}=\cdots=S_{k_{\alpha}}, S_{l_{1}}=\cdots=S_{l_{\alpha}}\right] \\
& =\sum_{x, y \in \mathbb{Z}^{d}} \sum_{p_{1} \leq \cdots \leq p_{2 \alpha} \leq n} \sum_{\epsilon: v(\delta) \geq 3} \mathbb{P}\left[S_{p_{1}}=x, S_{p_{2}}=x+\epsilon_{2} y, \ldots, S_{p_{2 \alpha}}=x+\epsilon_{2 \alpha} y\right] \\
& \leq \sum_{x, y \in \mathbb{Z}^{d}} \sum_{m_{0}, \ldots, m_{2 \alpha-1} \leq n} \sum_{\delta: v(\delta) \geq 3} \mathbb{P}\left(S_{m_{0}}=x\right) \mathbb{P}\left(S_{m_{0}, m_{1}}=\delta_{1} y\right) \cdots \mathbb{P}\left(S_{m_{2 \alpha-2}, m_{2 \alpha-1}}=\delta_{2 \alpha-1} y\right) \\
& =\sum_{y \in \mathbb{Z}^{d} m_{0}, \ldots, m_{2 \alpha-1} \leq n} \sum_{\delta: v(\delta) \geq 3} \mathbb{P}\left(S_{m_{0}, m_{1}}=\delta_{1} y\right) \cdots \mathbb{P}\left(S_{m_{2 \alpha-2}, m_{2 \alpha-1}}=\delta_{2 \alpha-1} y\right) .
\end{aligned}
$$

Summing over the free index $m_{0}$, it is clear that

$$
\begin{aligned}
& I_{n} \leq(n+1) \\
& \cdot \sum_{m_{1}, \ldots, m_{2 \alpha-1}} \sum_{y \in \mathbb{Z}^{d}} \sum_{\delta: v(\delta) \geq 3} \prod_{t=1}^{2 \alpha-1} \sup _{w} \mathbb{P}\left(S_{w, m_{t}}=\delta_{t} y\right) .
\end{aligned}
$$

For any $\delta=\left(\delta_{1}, \ldots, \delta_{2 \alpha-1}\right)$ with $v(\delta)=v$, exactly $u:=2 \alpha-1-v$ elements are equal to 0 , and therefore by Assumption (A) with $x=0$ we have

$$
\begin{aligned}
I_{n} \leq & C(n+1) \sum_{v=3}^{\alpha}\left[\sum_{i=0}^{n} \phi(i)\right]^{2 \alpha-1-v} \\
& \cdot \sum_{j_{1}, \ldots, j_{v}=0}^{n} \sum_{y \in \mathbb{Z}^{d}} \sum_{\delta^{\prime} \in\{-1,+1\}^{v}} \prod_{t=1}^{v} \sup _{w_{t}} \mathbb{P}\left(S_{w_{t}, j_{t}}=\delta_{t} y\right) .
\end{aligned}
$$

Letting $\left(\widetilde{S}_{n}\right)_{n \in \mathbb{N}_{0}}$ denote an independent copy of the random walk $\left(S_{n}\right)_{n \in \mathbb{N}_{0}}$ and assuming without loss of generality that $j_{1} \leq \cdots \leq j_{v}$, we have that for any $\delta \in\{-1,+1\}^{v}$

$$
\begin{aligned}
& \sum_{y \in \mathbb{Z}^{d}} \prod_{t=1}^{v} \sup _{w_{t}} \mathbb{P}\left(S_{w_{t}, j_{t}}=\delta_{t} y\right) \\
& \leq\left(\prod_{t=2}^{v-1} \sup _{y} \sup _{w_{t}} \mathbb{P}\left(S_{w_{t}, j_{t}}=y\right)\right) \\
& \cdot \sup _{w_{1}, w_{v}} \mathbb{P}\left(S_{w_{1}, j_{1}}-\delta_{v} \widetilde{S}_{w_{v}, j_{v}}=0\right) \leq\left[\prod_{t=2}^{v-1} \phi\left(j_{t}\right)\right] \\
& \cdot \phi\left(j_{1}+j_{v}\right) \leq \prod_{t=2}^{v} \phi\left(j_{t} \vee j_{1}\right) .
\end{aligned}
$$


Let $G_{n}:=\sum_{i=0}^{n} \phi(i)$. Since $\phi$ is nonincreasing we have that

$$
\begin{aligned}
\Delta_{n, v} & :=\sum_{0 \leq j_{1} \leq \cdots \leq j_{v} \leq n} \prod_{t=2}^{v} \phi\left(j_{t} \vee j_{1}\right) \\
& \leq \sum_{j_{v}=0}^{n} \phi\left(j_{v}\right) \sum_{0 \leq j_{1} \leq \cdots \leq j_{v-1} \leq n} \prod_{t=2}^{v-1} \phi\left(j_{t} \vee j_{1}\right) \\
& =G_{n} \Delta_{n, v-1},
\end{aligned}
$$

and iterating this procedure, for $v \geq 3$, we have that $\Delta_{n, v} \leq$ $\Delta_{n, 3} G_{n}^{v-3}$. Combining the two bounds and summing over $v=$ $3, \ldots, 2 \alpha-1$, we have that

$$
\begin{aligned}
I_{n} & \leq \sum_{v=3}^{2 \alpha-1} c(\alpha) n G_{n}^{2 \alpha-1-v} \Delta_{n, v} \leq c(\alpha) n G_{n}^{2 \alpha-1-v+v-3} \Delta_{n, 3} \\
& =c(\alpha) n G_{n}^{2 \alpha-4} \Delta_{n, 3},
\end{aligned}
$$

where $c(\alpha)$ is a constant depending only on $\alpha$.

Terms with $v=2$. Next we consider the sum $J_{n}$ over the terms with $v=2$, which occurs when, for some $j$, the indices $l_{1}, \ldots, l_{\alpha}$ all lie in $\left[k_{j}, k_{j+1}\right]$. Then it is easy to see that this sum $J_{n}$ is bounded above by

$$
\begin{aligned}
& J_{n} \leq C n \sup _{w_{0}, \ldots, w_{2 \alpha-1}} \sum_{m_{\alpha+1}, \ldots, m_{2 \alpha-2}=0}^{n} \prod_{r=\alpha+1}^{2 \alpha-2} \mathbb{P}\left(S_{w_{r}, m_{r}}=0\right) \\
& \cdot \sum_{m_{0}, \ldots, m_{\alpha}=0}^{n}\left[\prod_{t=1}^{\alpha-1} \mathbb{P}\left(S_{w_{t}, m_{t}}=0\right)\right]\left[\mathbb { P } \left(S_{w_{0}, m_{0}}+S_{w_{\alpha}, m_{\alpha}}\right.\right. \\
& \left.=0)-\mathbb{P}\left(S_{w_{0}, m_{0}}+\cdots+S_{w_{\alpha}, m_{\alpha}}=0\right)\right] \leq \operatorname{Cn}_{n}^{\alpha-2} \\
& \sup _{w_{0}, \ldots, w_{\alpha}} \sum_{m_{0}, \ldots, m_{\alpha}=0}^{n}\left[\prod_{t=1}^{\alpha-1} \mathbb{P}\left(S_{w_{t}, m_{t}}=0\right)\right] \\
& \cdot\left[\mathbb{P}\left(S_{w_{0}, m_{0}}+S_{w_{\alpha}, m_{\alpha}}=0\right)\right. \\
& \left.-\mathbb{P}\left(S_{w_{0}, m_{0}}+\cdots+S_{w_{\alpha}, m_{\alpha}}=0\right)\right] \\
& \leq \operatorname{CnG}_{n}^{\alpha-2} \sum_{m_{0}, \ldots, m_{\alpha}=0}^{n}\left[\prod_{t=1}^{\alpha-1} \phi\left(m_{t}\right)\right] \psi\left(m_{0}+m_{\alpha}, m_{1}\right. \\
& \left.+\cdots+m_{\alpha-1}\right) \leq \operatorname{C\alpha n} G_{n}^{\alpha-2} A_{\psi}\left(1+A_{\psi}\right)^{\alpha-2} \\
& \cdot\left(\sum_{m_{2}, \ldots, m_{\alpha-1}} \prod_{t=2}^{\alpha-1} \phi\left(m_{t}\right)\right) \sum_{m_{0}, m_{1}, m_{\alpha}} \phi\left(m_{1}\right) \psi\left(m_{0}+m_{\alpha}\right. \text {, } \\
& \left.m_{1}\right) \leq C \alpha A_{\psi}\left(1+A_{\psi}\right)^{\alpha-2} n G_{n}^{2 \alpha-4} \sum_{i, j, k=0}^{n} \phi(j) \psi(i \\
& +k, j) \text {. }
\end{aligned}
$$

The following corollary provides explicit bounds in the cases that are usually considered in the literature.
Corollary 5. Assume that the conditions of Proposition 4 are satisfied with $\phi(m)=T m^{-r}$ and $\psi(m, k)=T m^{-r-1}(k \wedge m)$. Then,

$$
\begin{aligned}
& \operatorname{var}\left(L_{n}(\alpha)\right) \\
& \leq c_{\alpha} T^{2 \alpha-2} \begin{cases}n^{2} \log (n)^{2 \alpha-4}, & \text { if } r=1, \\
n^{4-2 r}, & \text { if } 1<r<\frac{3}{2}, \\
n \log (n), & \text { if } r=\frac{3}{2}, \\
n, & \text { if } r>\frac{3}{2} .\end{cases}
\end{aligned}
$$

It is straightforward to see that Corollary 5 includes random walks with mean zero and finite second moment; for example, $d=2$ corresponds to $r=1$ and $d=3$ to $r=3 / 2$. Therefore several relevant results in $[3,7-13]$ are obtained as a special case of Corollary 5 and extended to the case of independent but not necessarily identically distributed variables, for example, by applying the local limit theorem, as conducted in [8].

Also when the random walk increment $X$ is in the domain of attraction of the one-dimensional symmetric Cauchy law $[13,14]$ or in the case of planar random walk with second moments $[3,7-9,11]$, it is well known that the conditions of Proposition 4 are satisfied with $\phi(m)=T / m$ and $\psi(m, k)=$ $\operatorname{Tm}^{-2}(k \wedge m)$.

However, we can do better for symmetric variables and show that condition (A) implies (B), which together with the comparison technique motivates the following results. For a real number $x$, we write $[x]$ for the integer part of $x$.

Proposition 6 (bounds via comparison with characteristic function of symmetric random variables). Let $X_{1}, X_{2}, \ldots$, be independent $\mathbb{Z}^{d}$-valued random variables and let $f_{i}(t):=$ $\mathbb{E} \exp \left(i t X_{i}\right)$. Assume that there exist a measurable function $f$ : $\Gamma \rightarrow[0,1]$ and a positive nonincreasing sequence $(\phi(m))_{m \in \mathbb{N}_{0}}$, such that

$$
\begin{aligned}
\left|1-f_{i}(t)\right| & \leq T f(t), \\
\left|f_{i}( \pm t)\right| & \leq f(t), \\
\int_{\Gamma} f(t)^{m} \mathrm{~d} t & \leq \phi(m),
\end{aligned}
$$

for all integers $i, m \geq 0$, all $t \in \Gamma$, and some positive constant $T$. Then there exists another positive constant $K=c(\alpha, d, T)$ such that

$$
\begin{aligned}
& \operatorname{var}\left(L_{n}(\alpha)\right) \\
& \leq K n\left(\sum_{i=0}^{n-1} \phi\left(\left[\frac{i}{2}\right]\right)\right)^{2 \alpha-4} \sum_{j=0}^{n} j \phi\left(\left[\frac{j}{2}\right]\right) \sum_{k=j}^{2 n} \phi\left(\left[\frac{k}{2}\right]\right) .
\end{aligned}
$$


Proof of Proposition 6. Using the notation of Proposition 4, for positive integers $a, u, b$, and $v$, with $a+u \leq b, \epsilon_{j}= \pm 1$, and any $x \in \mathbb{Z}^{d}$

$$
\begin{aligned}
& \mathbb{P}\left(S_{a, u}+\epsilon \cdot S_{b, v}=x\right) \\
& \leq \frac{1}{(2 \pi)^{d}} \int_{\Gamma} \prod_{j \in[a, a+u] \cup[b, b+v]}\left|f_{j}\left(\epsilon_{j} t\right)\right| \mathrm{d} t \\
& \quad \leq \frac{1}{(2 \pi)^{d}} \int_{\Gamma} f(t)^{u+v} \mathrm{~d} t \leq \frac{1}{(2 \pi)^{d}} \phi(u+v) .
\end{aligned}
$$

To find $\psi(u, v)$, notice that since $f(t) \geq 0$,

$$
\begin{aligned}
\phi(m) & \geq \int_{\Gamma} f(t)^{m}\left[1-f(t)^{m}\right] \mathrm{d} t \\
& =\sum_{j=0}^{m-1} \int_{\Gamma} f(t)^{m+j}(1-f(t)) \mathrm{d} t \\
& \geq m \int_{\Gamma} f(t)^{2 m}(1-f(t)) \mathrm{d} t=: m Q(2 m)
\end{aligned}
$$

whence $Q(m) \leq 2 \phi([m / 2]) / m$. Therefore,

$$
\begin{aligned}
\mid \mathbb{P} & \left(S_{a, u}=0\right)-\mathbb{P}\left(S_{a, u}+S_{b, 1}=0\right) \mid \\
& =\left|\frac{1}{(2 \pi)^{d}} \int_{\Gamma}\left[\prod_{j=a}^{a+u} f_{j}(t)\right]\left(1-f_{b+1}(t)\right) \mathrm{d} t\right| \\
& \leq C T \int_{\Gamma}|f(t)|^{u}|1-f(t)| \mathrm{d} t \leq \frac{C T \phi([u / 2])}{u} .
\end{aligned}
$$

A telescoping argument implies that

$$
\left|\mathbb{P}\left(S_{a, u}=0\right)-\mathbb{P}\left(S_{a, u}+S_{b, v}=0\right)\right| \leq C T \phi\left(\left[\frac{u}{2}\right]\right) \frac{v}{u}
$$

On the other hand for $u \leq v$ we can obtain a tighter bound through

$$
\begin{aligned}
& \mathbb{P}\left(S_{a, u}=0\right)-\mathbb{P}\left(S_{a, u}+S_{b, v}=0\right) \leq \mathbb{P}\left(S_{a, u}=0\right) \\
& \quad \leq \phi(u) .
\end{aligned}
$$

Combining the two bounds above it follows that (B) is satisfied with $\psi(u, v):=\phi([u / 2]) \min (u, v) / u$. Thus all conditions of Proposition 4 are satisfied and the result follows.

The following corollary allows for the case where $\phi(m)$ is regularly varying.

Corollary 7. Assume that the conditions of Proposition 6 are satisfied with $\phi(m)=h(m) m^{-r}, r \geq 1$, where $h(\cdot)$ is slowly varying at $\infty$. Then,

$$
\begin{aligned}
\operatorname{var}\left(L_{n}(\alpha)\right) \leq K \Delta_{n}(\alpha, \phi) & \begin{cases}n^{2}\left[\sum_{k=1}^{n} \frac{h(k)}{k}\right]^{2 \alpha-4}, & \text { for } r=1, \\
n^{4-2 r} h^{2}(n), & \text { for } 1<r<\frac{3}{2}, \\
n \sum_{k=1}^{n} \frac{h(k)^{2}}{k}, & \text { for } r=\frac{3}{2}, \\
n, & \text { for } r>\frac{3}{2} .\end{cases}
\end{aligned}
$$

Several results in $[3,7-13]$ are obtained as a special case of Corollary 7 and can be extended to dependent variables, for example, a random walk driven by a hidden Markov chain. In addition, following [2], we can construct a one-dimensional symmetric random walk with characteristic function $f(t)=$ $1-c|t|^{1 / r}+o\left(|t|^{1 / r}\right)$, where $r=2 / d$ for $d=2,3$ and $r=1 / 2$ for $d \geq 4$, whose asymptotic behaviour is similar to that of genuinely $d$-dimensional random walk.

The following example of genuinely 2 -dimensional recurrent walk with infinite variance was motivated by Spitzer [1, pp. 87].

Example 8. Let $X_{1}, X_{2}, \ldots$ be independent, identically distributed, $\mathbb{Z}^{2}$-valued random variables, such that $\mathbb{P}\left(\left|X_{1}\right|=\right.$ $k)=c /\left(k^{3} \log (k)^{g}\right)$, for $k \geq 4$ and $g \in[0,1)$. Let $\left(S_{n}\right)_{n \in \mathbb{N}_{0}}$ be the corresponding random walk in $\mathbb{Z}^{2}$. Then we have

$$
\begin{aligned}
& \operatorname{var}\left(L_{n}(\alpha)\right) \\
& \quad \leq c n^{2} \max \left\{[\log n]^{g}, \log \log n\right\}^{2 \alpha-4} \log n^{-2(1-g)},
\end{aligned}
$$

for $n \geq 10$. Under these assumptions we have that $\mathbb{P}\left(S_{n}=\right.$ $0) \leq c / n \log (n)^{1-g}$, which is in the critical range, where the random walk is recurrent, without second moment. To see why, we note that by a lengthy but straightforward calculation it can be shown that the characteristic function of $X$ satisfies (19) with

$$
\begin{aligned}
\phi(n) & =\frac{c}{n \log (\mathrm{e} \vee n)^{1-g}}, \\
f(t) & =\exp \left[-A|t|^{2} h\left(|t|^{2}\right)\right], \\
\text { where } h(r) & :=\left[1+\log \left(\frac{1}{r}\right)_{+}\right]^{1-g} .
\end{aligned}
$$

The sequence $\phi(m)$ is identified via Fourier inversion, polar coordinates, and a Laplace argument,

$$
\begin{aligned}
\int_{\Gamma} f(t)^{n} \mathrm{~d} t \leq & c \int_{0}^{1} \exp \left(-n r\left(1+\log \left(\frac{1}{r}\right)\right)^{1-g}\right) \\
& +O\left(\mathrm{e}^{-n}\right) \leq \frac{c}{n \log (\mathrm{e} \vee n)^{1-g}}=: \phi(n) .
\end{aligned}
$$

\subsection{Bounds for Identically Distributed Variables}

Proposition 9 (general upper bound for i.i.d.). Let $X, X_{1}, X_{2}, \ldots$, be independent, identically distributed, 
$\mathbb{Z}^{d}$-valued random variables. Suppose that for any $x \in \mathbb{Z}^{d}$ and all positive integers $a, u, b$, and $v$, with $a+u \leq b$, it holds that

$$
\mathbb{P}\left(S_{a, u} \pm S_{b, v}=x\right) \leq \phi(u+v),
$$

where $\{\phi(m)\}_{m \in \mathbb{N}_{0}}$ is a nonincreasing sequence. Then for some constant $K=c(\alpha)$ we have that

$$
\begin{aligned}
& \operatorname{var}\left(L_{n}(\alpha)\right) \\
& \quad \leq K n\left(\sum_{i=0}^{n-1} \phi(i)\right)^{2 \alpha-4} \sum_{j=0}^{n} j \phi(j) \sum_{k=j}^{[\alpha n]+1} \phi\left(\left[\frac{k}{\alpha}\right]\right) .
\end{aligned}
$$

Proof of Proposition 9. By inspecting the proof of Proposition 6 , we notice that we only need to bound the term $J_{n}$. Consider typical ordering

$$
0 \leq i_{1} \leq \cdots \leq i_{k} \leq j_{1} \leq \cdots \leq j_{\alpha} \leq i_{k+1} \leq \cdots \leq i_{\alpha} \leq n,
$$

and let us change variables to $\left(m_{0}, \ldots, m_{2 \alpha}\right)$ such that $m_{0}+$ $\cdots+m_{2 \alpha}=n$. Then the contribution to $J_{n}$ is given by

$$
\begin{aligned}
& \sum_{m_{0}, \ldots, m_{2 \alpha}} \prod_{\substack{j \neq k, k+\alpha \\
1 \leq j \leq 2 \alpha-1}} \mathbb{P}\left(S_{m_{j}}=0\right) \\
& \cdot\left[\mathbb{P}\left(S_{m_{k}+m_{k+\alpha}}=0\right)-\mathbb{P}\left(S_{m_{k}+\cdots+m_{k+\alpha}}=0\right)\right] .
\end{aligned}
$$

We keep $m_{j}$ fixed for $j \neq \alpha, k+\alpha$ and we sum over $m=$ $m_{k}+m_{k+\alpha}$ from 0 to some $M=M\left(n,\left\{m_{j}\right\}_{j \neq k, k+\alpha}\right)$. Then for given $m_{k+1}, \ldots, m_{k+\alpha-1}$, the term in the sum is

$$
\sum_{m=0}^{M}(m+1)\left[\mathbb{P}\left(S_{m}=0\right)-\mathbb{P}\left(S_{m+q}=0\right)\right],
$$

where $q:=m_{k+1}+\cdots+m_{k+\alpha-1}$. Then since $M \leq n-q$, it is an easy exercise to show that this sum is bounded above by

$$
\begin{aligned}
& \sum_{m=0}^{M}(m+1)\left[\mathbb{P}\left(S_{m}=0\right)-\mathbb{P}\left(S_{m+q}=0\right)\right] \\
& \leq \sum_{m=0}^{q-1}(m+1) \mathbb{P}\left(S_{m}=0\right)+q \mathbb{1}(n-q \geq q) \\
& \cdot \sum_{m=q}^{n-q} \mathbb{P}\left(S_{m}=0\right) \leq \sum_{m=0}^{\left(\alpha m^{*}\right) \wedge n}(m+1) \mathbb{P}\left(S_{m}=0\right) \\
& +\alpha m^{*} \sum_{m=m^{*}}^{n} \mathbb{P}\left(S_{m}=0\right),
\end{aligned}
$$

where $m^{*}=\max \left\{m_{k+1}, \ldots, m_{k+\alpha-1}\right\}$. The result follows by summing over all indices apart from $m^{*}$ and changing the order of summation.

\subsection{Proofs of Main Results}

Proof of Theorem 1. We apply a comparison argument found to be useful in many areas (e.g., Montgomery-Smith and Pruss [15], and Lefèvre and Utev [16]). More specifically we bound the quantity $\operatorname{var}\left(L_{n}\right)$ by the corresponding quantity for the symmetrised random walk.

Following Spitzer's argument we notice that with $f(t)=$ $\mathbb{E}\left[\exp \left(\mathrm{i} t \cdot X_{1}\right)\right]$

$$
\begin{gathered}
\mathbb{P}\left(S_{a, u}+\epsilon S_{b, v}=x\right) \leq c \int_{\Gamma}|f(t)|^{u}|f(-t)|^{v} \mathrm{~d} t \\
=c \int_{\Gamma}\left[|f(t)|^{2}\right]^{u / 2}\left[|f(-t)|^{2}\right]^{v / 2} \mathrm{~d} t .
\end{gathered}
$$

Since $|f(t)|^{2}$ is the characteristic function of a symmetric random variable in $\mathbb{Z}^{d}$, for some positive $\lambda$, we have 1 $|f(t)|^{2} \geq \lambda|t|^{2}$, and, hence,

$$
\begin{aligned}
\mathbb{P}\left(S_{a, u}+\epsilon S_{b, v}=x\right) & \leq c \int_{\Gamma} \exp \left[-\frac{\lambda(u+v)}{2}|t|^{2}\right] \mathrm{d} t \\
& \leq c(u+v)^{-d / 2} .
\end{aligned}
$$

The result follows from Proposition 9 applied with $\phi(m)=$ $m^{-d / 2}$. lemma.

The proof of Theorem 2 will be based on the following

Lemma 10. Assume $X, X_{1}, X_{2}, \ldots$ are independent, identically distributed, genuinely d-dimensional random variables such that $\mathbb{E}|X|^{2}=\infty$. Then there exists a monotone, slowly varying sequence $\left(h_{n}\right)_{n \in \mathbb{N}_{0}}$, such that $h_{n} \rightarrow 0$ as $n \rightarrow \infty$ and

$$
\sup _{x \in \mathbb{Z}^{d}} \mathbb{P}\left(S_{n}=x\right) \leq c_{d} \int_{\Gamma}\left|\mathbb{E} e^{i t \cdot X}\right|^{n} \mathrm{~d} t \leq h_{n} n^{-d / 2} .
$$

Proof of Lemma 10. Without loss of generality we assume that $X$ is symmetric. Let $\sigma_{e, L}:=\mathbb{E}\left[(e \cdot X)^{2} \mathbb{\mathbb { }}(|X| \leq L)\right]$. Following Spitzer, since $X$ is genuinely $d$-dimensional, we may assume that there exist positive constants $c, W$, such that for any unit vector $|e|=1$ we have that $\sigma_{e, W} \geq c$ and $1-f(t) \geq c|t|^{2}$ for all $t \in \Gamma$. Let $\lambda_{d}$ be the $d$-dimensional Lebesgue measure on $\mathbb{R}^{d}$ and $\mu_{d}$ the Lebesgue-Haar measure on $S^{d-1}:=\{e \in \Gamma$ : $|e|=1\}$. Notice that since $\mathbb{E}|X|^{2}=\infty$, for any $K$, we have $\mu_{d}\left\{e: \sigma_{e, \infty}<K\right\}=0$.

Fix a small positive $x$ such that $\sqrt{c / x} \geq 2 W$, and for any $\epsilon>0$ let $K=K(\epsilon)=\epsilon^{-d / 2}$. Then there exists $L=L(\epsilon)>0$ small enough so that $\mu_{d}\left\{e: \sigma_{e, L}<K\right\} \leq \epsilon^{d / 2}$. We partition $S^{d-1}$ in two sets

$$
\begin{aligned}
& A_{L, K}=\left\{e \in S_{d}: \sigma_{e, L} \geq K\right\}, \\
& \bar{A}_{L, K}=\left\{e \in S_{d}: \sigma_{e, L}<K\right\},
\end{aligned}
$$

so that, for any direction $e \in \bar{A}_{L, K}$,

$$
\begin{aligned}
& \{z \in \mathbb{R}: 1-f(z e) \leq x\} \subseteq\left\{z: c z^{2} \leq x\right\} \\
& \quad \subseteq\left\{z:|z| \leq \sqrt{\frac{x}{c}}\right\} .
\end{aligned}
$$


Hence, using $d$-dimensional spherical coordinates,

$$
\begin{aligned}
\lambda_{d} & \left\{(z, e) \in \mathbb{R} \times \bar{A}_{L, K}: 1-f(e z) \leq x\right\} \\
& \leq \mu_{d}\left\{\bar{A}_{L, K}\right\}\left(\frac{x}{c}\right)^{d / 2}\left(\frac{1}{d}\right) \leq \epsilon^{d / 2}\left(\frac{x}{c}\right)^{d / 2}\left(\frac{1}{d}\right) .
\end{aligned}
$$

On the other hand, for any $t$,

$$
\begin{aligned}
1-f(t) & =2 \sum_{k \in Z^{d}} \sin \left(\frac{[t \cdot k]}{2}\right)^{2} P(X=k) \\
& \geq\left(\frac{1}{4}\right) E\left[(t \cdot X)^{2} I\left(|t \cdot X| \leq \frac{1}{2}\right)\right] \\
& =\left(\frac{|t|^{2}}{4}\right) \sigma_{t /|t|, 1 / 2|t|} .
\end{aligned}
$$

Now, assume that $\sqrt{c / x} \geq 2 L$. Then for any direction $e \in$ $A_{L, K}$, by choice of $x$ and since $\sigma_{e, L}$ is increasing in $L$, for $c z^{2} \leq$ $1-f(e z) \leq x$ or $|z| \leq \sqrt{x / c}$, it must be the case that

$$
\begin{aligned}
x & \geq 1-f(e z) \geq\left(\frac{z^{2}}{4}\right) \sigma_{e, 1 / 2 z} \geq\left(\frac{z^{2}}{4}\right) \sigma_{e, L} \\
& \geq\left(\frac{z^{2}}{4}\right) K,
\end{aligned}
$$

implying that, on the set $A_{L, K}$, it must be that $|z| \leq 2 \sqrt{x / K}$. Changing to $d$-dimensional polar coordinates, we find that

$$
\begin{aligned}
& \lambda_{d}\left\{(z, e) \in \mathbb{R} \times A_{L, K}: 1-f(e z) \leq x\right\} \\
& \quad \leq \int_{A_{L, K}} \int_{0}^{\sqrt{4 x / K}} r^{d-1} \mathrm{~d} r \mathrm{~d} e \leq C_{d} \epsilon^{d / 2} x^{d / 2} .
\end{aligned}
$$

Overall, for $x \leq c / 4 L^{2}, \lambda_{d}\{t: 1-f(t) \leq x\} \leq c_{d}(x \epsilon)^{d / 2}$, and hence $\{t \in \Gamma: 1-f(t) \leq x\}$ has Lebesgue measure $o\left(x^{d / 2}\right)$.

Let $F(x)$ be the cumulative distribution function of the random variable $\log (1 / f(\cdot))$ defined on the probability space $\Gamma$ with normalised Lebesgue measure. Then $F$ is continuous at $x=0$ and supported on $\mathbb{R}^{+}$. Moreover, we have that $F(x)=o\left(x^{d / 2}\right)$ as $x \downarrow 0$. Therefore, for some positive sequence $\left(\epsilon_{n}\right)_{n \in \mathbb{N}_{0}}$ with $\epsilon_{n} \rightarrow 0$, we have that

$$
\begin{aligned}
\frac{1}{(2 \pi)^{2}} \int_{\Gamma} f(t)^{n} \mathrm{~d} t & =\int_{0}^{\infty} \mathrm{e}^{-n x} \mathrm{~d} F(x) \\
& =n \int_{0}^{\infty} \mathrm{e}^{-n x} F(x) \mathrm{d} x \leq n^{-d / 2} \epsilon_{n}
\end{aligned}
$$

It remains to show that there exists a positive, monotone, slowly varying sequence $\left(h_{n}\right)_{n \in \mathbb{N}_{0}}$, such that $\epsilon_{n} \leq h(n) \rightarrow 0$ as $n \rightarrow \infty$. Let $\delta_{n}=\sup _{j \geq n} \epsilon_{j}$ and $a_{0}:=0$ and for $n \geq 1$ define $a_{n}$ recursively by $a_{n}=\min \left(2 a_{2^{r-1}}, 1 / \delta_{n}\right)$, for $2^{r-1}<n \leq 2^{r}$, so that $a_{n} \rightarrow \infty$ is monotone, $a_{2^{r}} \leq 2 a_{2^{r-1}}$ implying that $a_{2 n} \leq$ $4 a_{n}$, and $1 / a_{n} \geq \delta_{n} \geq \epsilon_{n}$. Finally, take $h_{n}:=1 / \max \left(a_{0}, \log a_{n}\right)$.
Proof of Theorem 2. Assume that $\mathbb{E}|X|^{2}=\infty$ and $d=2$ or $d=$ 3. Then, by Lemma 10 there exists a slowly varying sequence $h_{n} \rightarrow 0$ as $n \rightarrow \infty$ such that $\int_{\Gamma}|\mathbb{E} \exp (\mathrm{i} t \cdot X)|^{n} \mathrm{~d} t \leq h_{n} n^{-d / 2}$. Applying Corollary 7 with $r=1$ and $r=3 / 2$ we, respectively, find that

$$
\begin{aligned}
& \operatorname{var}\left(L_{n}(\alpha)\right) \\
& \leq \begin{cases}K n^{2}\left(\sum_{k=1}^{n} \frac{h(k)}{k}\right)^{2 \alpha-4}=o\left(n^{2}(\log n)^{2 \alpha-4}\right), & \text { for } d=2, \\
K n\left(\sum_{k=1}^{n} \frac{h(k)^{2}}{k}\right)=o(n \ln n), & \text { for } d=3 .\end{cases}
\end{aligned}
$$

Finally assume that $\mathbb{E}|X|^{2}<\infty$ and $E[X]=\mu \neq 0$. Then $\mathbb{P}\left(S_{n}=0\right)=\mathbb{P}\left(S_{n}^{\prime}=-n \mu\right)$ whence it follows that $\mathbb{P}\left(S_{n}=\right.$ $0)=o\left(n^{-d / 2}\right)$ (see, e.g., [17, Theorem 2.3.10]). Then inspecting the proof of Proposition 4, one can readily obtain the desired bound for the $J_{n}$ term, while with slight modification the bound for the $I_{n}$ term also follows.

Note that for $d=1$ the situation is much simpler since then $\operatorname{var}\left(L_{n}^{\mathrm{SRW}}(\alpha)\right) \sim C\left[\mathbb{E} L_{n}^{\mathrm{SRW}}(\alpha, d)\right]^{2}$ and if $\mathbb{E}|X|^{2}=\infty$ or $\mathbb{E}[X] \neq 0, \mathbb{E} L_{n}^{\mathrm{SRW}}(\alpha, d)=o\left(n^{(1+\alpha) / 2}\right)$.

Proof of Theorem 3. We first give the proof for the case $d=1$. As in the proof of Proposition 4 we begin from expression (10) and define the sequences $p_{i}$ and $\delta_{i}$ for $i=1, \ldots, 2 \alpha-1$, and the quantity $v(\delta)=\sum_{i=1}^{2 \alpha-1}\left|\delta_{i}\right|$. Recall that $v(\delta)$ measures the interlacement of the two sequences $k_{1}, \ldots, k_{\alpha}$ and $l_{1}, \ldots, l_{\alpha}$. For example, $v(\delta)=1$ occurs when either $k_{\alpha} \leq l_{1}$ or $l_{\alpha} \leq$ $k_{1}$, in which case the contribution vanishes by the Markov property. On the other hand $v(\delta)=2$ when, for example, $l_{1}, \ldots, l_{\alpha} \in\left[k_{i}, k_{i+1}\right]$ for some $i$. Finally $v(\delta)=3$ occurs when, for example,

$$
\begin{aligned}
k_{1} & \leq \cdots \leq k_{r} \leq l_{1} \leq \cdots \leq l_{s} \leq k_{r+1} \leq \cdots \leq k_{\alpha} \leq l_{s+1} \\
& \leq \cdots \leq l_{\alpha} \leq n
\end{aligned}
$$

From the proof of Proposition 4 , and using the bound $\mathbb{P}\left(S_{n}=\right.$ $0) \leq c / n$, the terms of the sum are bounded above by $n^{2} \log (n)^{2 \alpha-1-v(\delta)}$, and thus the leading term appears when either $v(\delta)=2,3$, with other terms giving strictly lower order. We will therefore analyze these two situations in detail in order to derive the exact asymptotic constants. When $v=3$, the two terms in the difference individually give the correct order and will be treated by the classical Tauberian theory. However for $v=2$, the two terms only give the correct order when considered together. This however forbids the use of Karamata's Tauberian theorem since the monotonicity restriction would require roughly that $X_{i}$ is symmetric. Thus the complex Tauberian approach, as developed in [13], is required to justify the answer.

Case $1(v(\delta)=3)$. Assume that part of the sequence $\mathbf{1}=$ $\left\{l_{1}, \ldots, l_{\alpha}\right\}$ lies between $k_{r}$ and $k_{r+1}$ and the rest between $k_{s}$ and $k_{s+1}$. Then using the change of variables 


$$
\begin{gathered}
i_{1}=m_{0}, \\
i_{2}=m_{0}+m_{1}, \\
\vdots \\
i_{r}=m_{0}+\cdots+m_{r-1} \\
j_{1}=m_{0}+\cdots+m_{r}, \\
j_{2}=m_{0}+\cdots+m_{r+1}, \\
\vdots \\
j_{s}=m_{0}+\cdots+m_{r+s-1}, \\
i_{r+1}=m_{0}+\cdots+m_{r+s}, \\
i_{r+2}=m_{0}+\cdots+m_{r+s+1}, \\
\vdots \\
j_{s+2}=m_{0}+\cdots+m_{\alpha+s+1}, \\
\vdots \\
j_{\alpha}=m_{0}+\cdots+m_{\alpha+s-1}, \\
+\cdots+m_{\alpha+s},
\end{gathered}
$$

we rewrite the positive term in (10) as

$$
\begin{aligned}
& a(n) \\
& \quad=\sum \mathbb{P}\left[S\left(i_{1}\right)=\cdots=S\left(i_{\alpha}\right) ; S\left(j_{1}\right)=\cdots=S\left(j_{\alpha}\right)\right] \\
& \quad=\sum_{m_{0}, \ldots, m_{2 \alpha-1}}\left[\prod_{j=1}^{2 \alpha-1} \mathbb{P}\left(S_{m_{j}}=0\right)\right] \\
& \quad \cdot \mathbb{P}\left(S_{m_{r}}+S_{m_{r+s}}^{\prime}=S_{m_{r+s}}^{\prime}+S_{m_{\alpha+s}}^{\prime \prime}=0\right) .
\end{aligned}
$$

Notice that from [13] we have that $\sum_{n \geq 0} \lambda^{n} \mathbb{P}\left(S_{n}=0\right) \sim$ $\log (1 /(1-\lambda)) / \pi \gamma$. Let

$$
\begin{aligned}
a(\lambda) & =(1-\lambda)^{-3}[-\log (1-\lambda)]^{2 \alpha-4}, \\
c_{\gamma} & =(\pi \gamma)^{-2 \alpha+4}
\end{aligned}
$$

$$
\begin{aligned}
& (2 \pi)^{-\alpha-s+r} \int_{-\pi}^{\pi} \ldots \int_{-\pi}^{\pi}(1-\lambda \phi(x))^{-1}(1-\lambda \phi(x) \phi(y))^{-1}(1-\lambda \phi(y))^{-1} \\
& \cdot\left[\prod_{j=r+1}^{r+s-1} \prod_{k=r+s+1}^{\alpha+s-1}\left(1-\lambda \phi(x) \phi\left(t_{j}\right)\right)^{-1}\left(1-\lambda \phi(y) \phi\left(t_{k}\right)\right)^{-1} \mathrm{~d} t_{j} \mathrm{~d} t_{k}\right] \mathrm{d} x \mathrm{~d} y \sim(\pi \gamma)^{-\alpha-s+r}(1-\lambda)^{-1} \\
& \cdot \log \left(\frac{1}{1-\lambda}\right)^{\alpha-r+s-2} \frac{\pi^{2}}{6} .
\end{aligned}
$$


Then, we have $\sum_{n} \lambda^{n} b(n) \sim\left(\pi^{2} / 6(\pi \gamma)^{2 \alpha-2}\right) a(\lambda)$, whence the Tauberian theorem implies that $a(n)-b(n)$ $n^{2} \log (n)^{2 \alpha-4} / 24 \pi^{2 \alpha-4} \gamma^{2 \alpha-2}$. Most importantly we see that the lengths and locations of the chains, $r$ and $s$, do not affect the asymptotic behaviour. Noting that if $1 \leq r, s \leq \alpha-1$, we can partition $2 \alpha=r+s+(\alpha-r)+(\alpha-s)$ in $(\alpha-1)^{2}$ ways, and thus overall the total contribution from terms with $v=3$ is

$$
\left[\frac{(\alpha !(\alpha-1))^{2}}{12 \pi^{2 \alpha-4} \gamma^{2 \alpha-2}}\right] n^{2} \log (n)^{2 \alpha-4} .
$$

Case $2(v(\delta)=2)$. The typical term $c(n)$ was introduced in (33) in the proof of Proposition 9. Now we let $\lambda \in \mathbb{C}$, with $|\lambda|<1$. By lengthy but direct calculations we can derive an expression of the form

$$
\sum_{n} \lambda^{n} c(n)=\frac{\alpha-1}{(\gamma \pi)^{2 \alpha-2}} a(\lambda)+o(a(\lambda)), \quad \lambda \longrightarrow 1 .
$$

The approach developed in [13] can then be used to bound the error terms and show that $c(n) \sim[(\alpha-$ 1) $\left./ 2(\gamma \pi)^{2 \alpha-2}\right] n^{2} \log (n)^{2 \alpha-4}$.

Finally taking into account the fact that $l_{1}, \ldots, l_{\alpha}$ can be in any of the $\alpha-1$ intervals $\left[k_{i}, k_{i+1}\right]$, for $i=1, \ldots, \alpha-1$, the result follows the overall contribution of terms with $v(\delta)=2$

$$
\frac{(\alpha-1)^{2}}{2(\gamma \pi)^{2 \alpha-2}} n^{2} \log (n)^{2 \alpha-4}
$$

The case for $d=2$ is very similar, so we move on to the case $d=3$.

Case 3 ( $d=3$ and $\alpha=2)$. Using the same notation as before, we have three terms to consider $a(n), b(n)$, and $c(n)$. We first consider $c(n)$. Letting $K:=\epsilon / \sqrt{1-\lambda}$ and using the usual power series construction and spherical coordinates

$$
\sum_{n} \lambda^{n} c(n)=(1-\lambda)^{-2}(2 \pi)^{-6}
$$

$$
\begin{aligned}
& \cdot \iint_{\Gamma^{3} \times J^{3}} \frac{\lambda f(y)(1-f(x)) \mathrm{d} x \mathrm{~d} y}{(1-\lambda f(x))^{2}(1-\lambda f(y))(1-\lambda f(x) f(y))} \\
& \sim 2(2 \pi)^{-4}|\Sigma|^{-1}(1-\lambda)^{-2} \\
& \cdot \iint_{0}^{K} \frac{r^{4} s^{2} \mathrm{~d} r \mathrm{~d} s}{\left(1+r^{2}\right)^{2}(1+s)^{2}\left(1+r^{2}+s^{2}\right)} \sim 2(2 \pi)^{-4}|\Sigma|^{-1} \\
& \cdot \frac{\pi}{2}(1-\lambda)^{-2} \log \left(\frac{1}{1-\lambda}\right)=: \kappa_{1}(1-\lambda)^{-2} \log \left(\frac{1}{1-\lambda}\right),
\end{aligned}
$$

and thus $c(n) \sim \kappa_{1} n \log n$, where $\kappa_{1}>0$, where the answer can be justified following [13].

The term $a(n)-b(n)$ is trickier to compute. As usual we consider the power series

$$
\begin{aligned}
& \sum_{n \geq 0} \lambda^{n}(a(n)-b(n))=(1-\lambda)^{-2}(2 \pi)^{-6} \\
& \quad \cdot \iint_{B(\epsilon)} \frac{\mathrm{d} x \mathrm{~d} y}{(1-\lambda f(x))(1-\lambda f(y))(1-\lambda f(x+y))} \\
& \quad-(1-\lambda)^{-2}(2 \pi)^{-6} \\
& \quad \cdot \iint_{B(\epsilon)} \frac{\mathrm{d} x \mathrm{~d} y}{(1-\lambda f(x))(1-\lambda f(y))(1-\lambda f(x) f(y))} \\
& \quad=(1-\lambda)^{-2}(2 \pi)^{-6}\left(I_{1}(\lambda)-I_{2}(\lambda)\right) .
\end{aligned}
$$

Let $A \in[-1,1]$ be the cosine of the angle between $x$ and $y$, which in spherical coordinates is

$$
\begin{aligned}
A= & A\left(\theta_{1}, \theta_{2}, \phi_{1}, \phi_{2}\right) \\
= & \cos \left(\phi_{1}-\phi_{2}\right) \sin \left(\theta_{1}\right) \sin \left(\theta_{2}\right) \\
& +\cos \left(\theta_{1}\right) \cos \left(\theta_{2}\right) .
\end{aligned}
$$

Then as $0<\lambda \uparrow 1$, using the expansion (6)

$$
\begin{aligned}
I_{1}(\lambda) & \sim|\Sigma|^{-1} \int_{r, s=0}^{\epsilon} \int_{\phi_{1,2}=0}^{2 \pi} \int_{\theta_{1}, \theta_{2}=0}^{\pi} \frac{r^{2} s^{2} \sin \left(\theta_{1}\right) \sin \left(\theta_{2}\right) \mathrm{d} \theta_{1} \mathrm{~d} \theta_{2} \mathrm{~d} \phi_{1} \mathrm{~d} \phi_{2} \mathrm{~d} r \mathrm{~d} s}{\left(1-\lambda+\lambda r^{2}\right)\left(1-\lambda+\lambda s^{2}\right)\left[1-\lambda+\lambda\left(r^{2}+s^{2}+2 A r s\right)\right]} \\
& =|\Sigma|^{-1} \int_{\theta_{1}, \theta_{2}=0}^{\pi} \int_{\phi_{1}, \phi_{2}=0}^{2 \pi} \int_{r, s=0}^{K} \frac{\sin \left(\theta_{1}\right) \sin \left(\theta_{2}\right) r^{2} s^{2} \mathrm{~d} s \mathrm{~d} r \mathrm{~d} \phi_{1} \mathrm{~d} \phi_{2} \mathrm{~d} \theta_{1} \mathrm{~d} \theta_{2}}{\left(1+r^{2}\right)\left(1+s^{2}\right)\left[1+r^{2}+s^{2}+2 A r s\right]} \\
& \sim|\Sigma|^{-1} \log (K) \int_{\theta_{1}, \theta_{2}=0}^{\pi} \int_{\phi_{1}, \phi_{2}=0}^{2 \pi} \sin \left(\theta_{1}\right) \sin \left(\theta_{2}\right) \frac{\arccos \left(A\left(\theta_{1}, \theta_{2}, \phi_{1}, \phi_{2}\right)\right)}{\sqrt{1-A\left(\theta_{1}, \theta_{2}, \phi_{1}, \phi_{2}\right)^{2}}} \mathrm{~d} \phi_{1} \mathrm{~d} \phi_{2} \mathrm{~d} \theta_{1} \mathrm{~d} \theta_{2} .
\end{aligned}
$$

The other integral is slightly easier

$$
\begin{aligned}
& I_{2}(\lambda) \sim|\Sigma|^{-1} \frac{\pi}{2} \log K \\
& \quad \cdot \int_{\theta_{1}, \theta_{2}=0}^{\pi} \int_{\phi_{1}, \phi_{2}=0}^{2 \pi} \sin \left(\theta_{1}\right) \sin \left(\theta_{2}\right) \mathrm{d} \phi_{1} \mathrm{~d} \phi_{2} \mathrm{~d} \theta_{1} \mathrm{~d} \theta_{2},
\end{aligned}
$$

and thus overall we must have that

$$
\begin{gathered}
\left(I_{1}-I_{2}\right)(\lambda) \sim \frac{1}{2}(2 \pi)^{-6}|\Sigma|^{-1}(1-\lambda)^{-2} \log \left(\frac{1}{1-\lambda}\right) \\
\cdot \int_{\theta_{1}, \theta_{2}=0}^{\pi} \int_{\phi_{1}, \phi_{2}=0}^{2 \pi}\left[\frac{\arccos (A)}{\sqrt{1-A^{2}}}-\frac{\pi}{2}\right] \sin \left(\theta_{1}\right)
\end{gathered}
$$




$$
\begin{aligned}
& \cdot \sin \left(\theta_{2}\right) \mathrm{d} \phi_{1} \mathrm{~d} \phi_{2} \mathrm{~d} \theta_{1} \mathrm{~d} \theta_{2}=: \kappa_{2}(1 \\
& -\lambda)^{-2} \log \left(\frac{1}{1-\lambda}\right)
\end{aligned}
$$

whence it follows that $\operatorname{var}\left(L_{n}(2)\right) \sim\left(\kappa_{1}+\kappa_{2}\right) n \log n$.

To prove the last claim let $S_{n}^{\prime}=X_{1}^{\prime}+\cdots+X_{n}^{\prime}$ be another random walk, independent of $S_{n}$, such that its characteristic function $f^{\prime}(t)=\mathbb{E}\left[\exp \left(\mathrm{it} X_{i}^{\prime}\right)\right]$ also satisfies the expansion (6). Then using [13, Lemma 3.1], one can adapt the proof of [13, Theorem 2.1] to show that $L_{n}^{\prime}(\alpha)=L_{n}(\alpha)+o\left(L_{n}(\alpha)\right)$.

\section{Competing Interests}

The authors declare that they have no competing interests.

\section{References}

[1] F. Spitzer, Principles of Random Walk, Springer, 1976.

[2] H. Kesten and F. Spitzer, "A limit theorem related to a new class of self-similar processes," Zeitschrift für Wahrscheinlichkeitstheorie und Verwandte Gebiete, vol. 50, no. 1, pp. 5-25, 1979.

[3] E. Bolthausen, "A central limit theorem for two-dimensional random walks in random sceneries," The Annals of Probability, vol. 17, no. 1, pp. 108-115, 1989.

[4] A. N. Borodin, "A limit theorem for sums of independent random variables defined on a recurrent random walk," Doklady Akademii Nauk SSSR, vol. 246, no. 4, pp. 786-787, 1979.

[5] D. C. Brydges and G. Slade, "The diffusive phase of a model of self-interacting walks," Probability Theory and Related Fields, vol. 103, no. 3, pp. 285-315, 1995.

[6] J. Gärtner and R. Sun, "A quenched limit theorem for the local time of random walks on $\mathrm{Z}^{2}$," Stochastic Processes and Their Applications, vol. 119, no. 4, pp. 1198-1215, 2009.

[7] J. Černý, "Moments and distribution of the local time of a two-dimensional random walk," Stochastic Processes and their Applications, vol. 117, no. 2, pp. 262-270, 2007.

[8] G. F. Lawler, Intersections of Random Walks, Birkhäuser, Boston, Mass, USA, 1991.

[9] T. M. Lewis, "A law of the iterated logarithm for random walk in random scenery with deterministic normalizers," Journal of Theoretical Probability, vol. 6, no. 2, pp. 209-230, 1993.

[10] M. Becker and W. König, "Moments and distribution of the local times of a transient random walk on $\mathbb{Z}^{d}$," Journal of Theoretical Probability, vol. 22, no. 2, pp. 365-374, 2009.

[11] F. Castell, N. Guillotin-Plantard, and F. Pène, "Limit theorems for one and two-dimensional random walks in random scenery," Annales de l'Institut Henri Poincaré, Probabilités et Statistiques, vol. 49, no. 2, pp. 506-528, 2013.

[12] X. Chen, Random Walk Intersections, vol. 157 of Mathematical Surveys and Monographs, American Mathematical Society, Providence, RI, USA, 2010.

[13] G. Deligiannidis and S. A. Utev, "Computation of the asymptotics of the variance of the number of self-intersections of stable random walks using the Wiener-Darboux theory," Siberian Mathematical Journal, vol. 52, no. 4, pp. 809-822, 2011.

[14] G. Deligiannidis and S. Utev, "An asymptotic variance of the self-intersections of random walks," 2010.
[15] S. J. Montgomery-Smith and A. R. Pruss, "A comparison inequality for sums of independent random variables," Journal of Mathematical Analysis and Applications, vol. 254, no. 1, pp. 35-42, 2001.

[16] C. Lefèvre and S. Utev, "Exact norms of a Stein-type operator and associated stochastic orderings," Probability Theory and Related Fields, vol. 127, no. 3, pp. 353-366, 2003.

[17] G. F. Lawler and V. Limic, Random Walk: A Modern Introduction, vol. 123 of Cambridge Studies in Advanced Mathematics, Cambridge University Press, Cambridge, UK, 2010. 


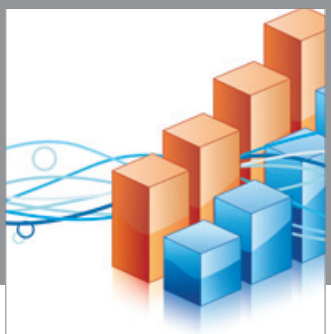

Advances in

Operations Research

vatem alat4

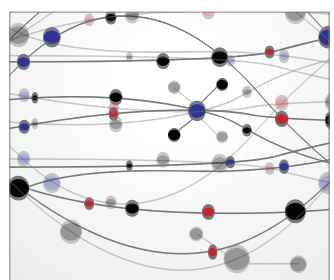

\section{The Scientific} World Journal
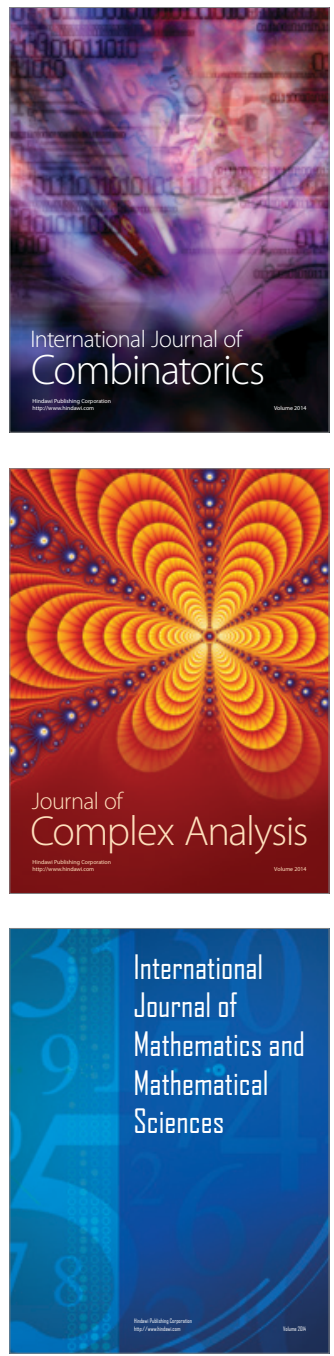
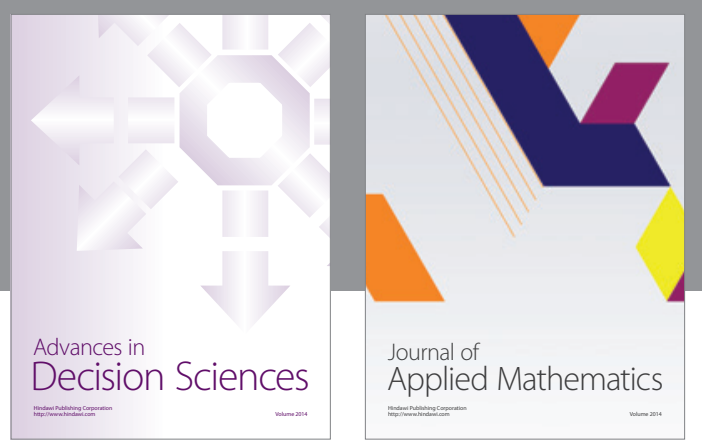

Algebra

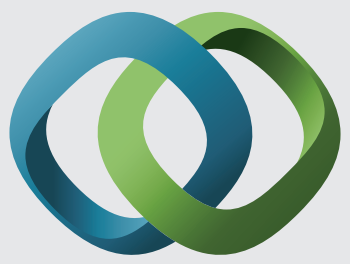

\section{Hindawi}

Submit your manuscripts at

http://www.hindawi.com
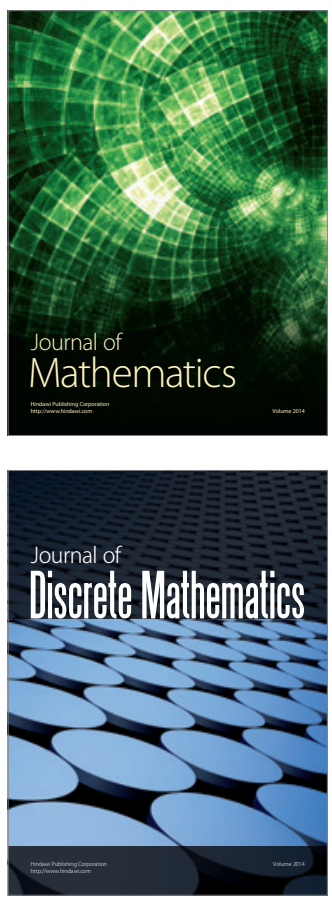

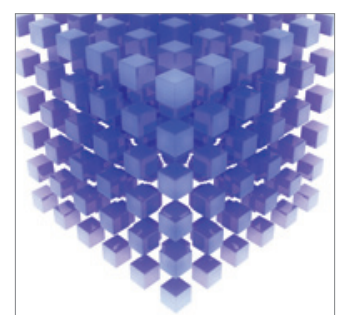

Mathematical Problems in Engineering
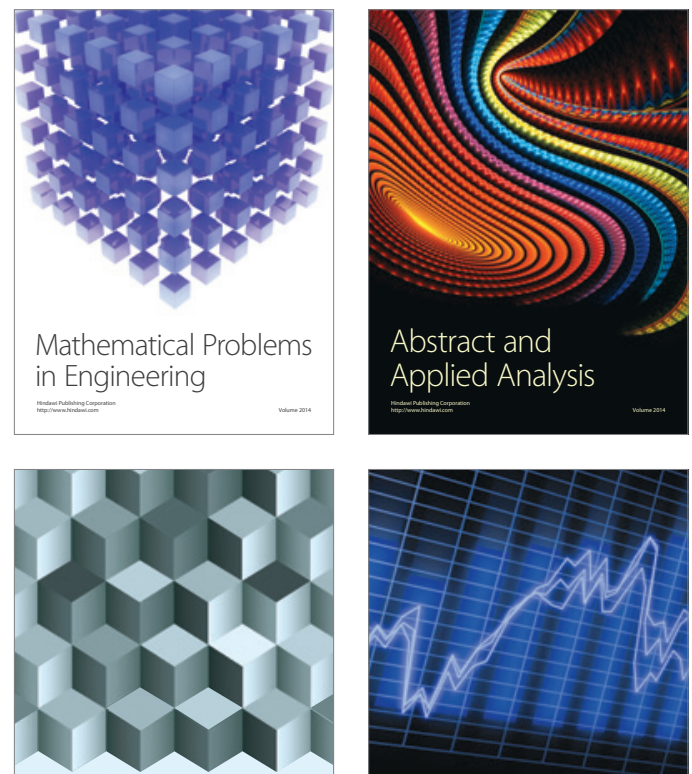

Journal of

Function Spaces

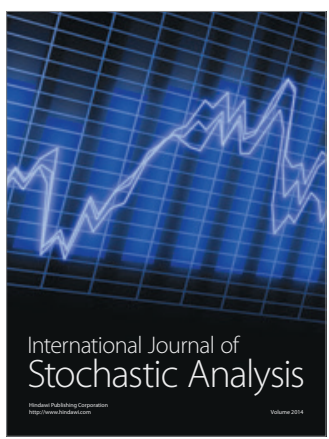

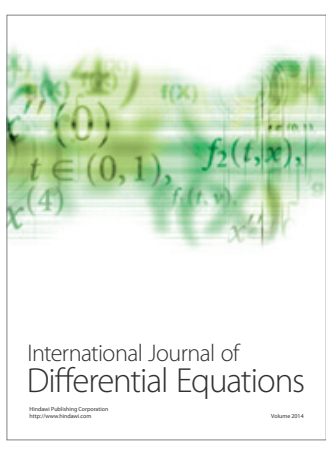
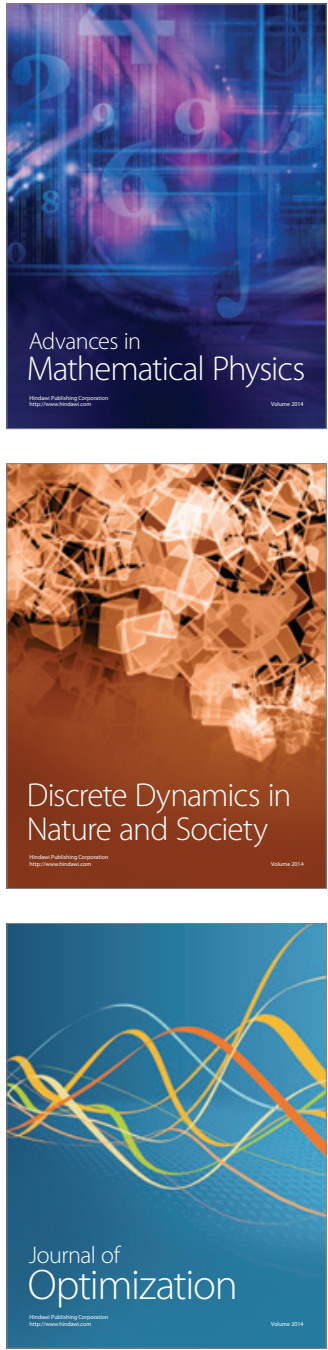